\title{
Correlations between COPD Assessment Test and Modified British Medical Research Council Scoring and Degree of Airflow Limitation
}

\author{
Dian Nuryanda, ${ }^{1}$ Hendarsyah Suryadinata, ${ }^{2}$ Ambrosius Purba ${ }^{3}$ \\ ${ }^{1}$ Faculty of Medicine Universitas Padjadjaran, ${ }^{2}$ Department of Internal Medicine Faculty of \\ Medicine, Universitas Padjadjaran/Dr. Hasan Sadikin General Hospital Bandung, ${ }^{3}$ Department of \\ Physiology Faculty of Medicine, Universitas Padjadjaran
}

\begin{abstract}
Background: Chronic Obstructive Pulmonary Disease (COPD) Assessment Test (CAT) and Modified British Medical Research Council (MMRC) dyspnea scale are tools used to assess health status in COPD patients, while Forced Expiratory Volume in one second (FEV1) may represent the degree of airflow limitation. Assessment of COPD patients is rarely performed comprehensively. This study aimed to determine the relationships of CAT and MMRC dyspnea scale and the degree of airflow limitation in COPD patients.

Methods: The study was conducted from September until October 2014 after obtaining approval from the Hasan Sadikin General Hospital Ethics Committee. This observational analytic study was performed on 77 medical records of COPD patients at Hasan Sadikin General Hospital. Patients' medical records included in this study were those with spirometry measurements results, CAT and MMRC dyspnea scale scoring. Data normality was analyzed using Kolmogorov-Smirnov Test. Pearson's Correlation was used for parametric variables. Spearman's correlation was used for non-parametric variables.
\end{abstract}

Results: Results showed Pearson's correlation of CAT and FEV1 ( $p$ value $=0.035$ and $r=0.240$ ), and Spearman's correlation of MMRC dyspnea scale and FEV1 ( $\mathrm{p}$ value $=0.198$ and $\mathrm{r}=0.148$ ).

Conclusions: CAT scoring is significantly correlated with the degree of airway limitation. MMRC dyspnea scale is not significantly correlated with the degree of airway limitation.

Keywords: Chronic Obstructive Pulmonary Disease (COPD), COPD Assessment Test (CAT), degree of airway limitation, Modified British Medical Research Council (MMRC)

\section{Introduction}

The World Health Organization (WHO) categorizes Chronic Obstructive Pulmonary Disease (COPD) as the third leading cause of death in non-communicable disease. The burden of COPD is predicted to increase in the coming years. ${ }^{1,2}$ The goal of COPD assessment is to determine the disease severity, the impact towards patient's health status, and risks that may arise such as exacerbations, hospital admissions, or even death. Eventually, the goal of this assessment is to achieve a guide therapy for COPD patients. Airway obstruction in COPD can be assessed by spirometry measurement. While the patient's health status can be assessed by tools such as COPD Assessment Test (CAT) and Modified Medical Research Council (MMRC) dyspnea scale. ${ }^{1}$
Furthermore, Forced Expiratory Volume in one second (FEV1) after bronchodilator administration shows the degree of obstruction in COPD and represents the disease severity. CAT is a valid and reliable tool to assess health status in COPD patients; it consists of eight items that assess the patient's general condition. CAT is an easy and applicable tool to use. ${ }^{3}$ MMRC dyspnea scale is developed to help in determining the degree of dyspnea on emphysema patient clinically, it assess patient's limitation in daily activity. ${ }^{4}$

The routine examination on COPD patients at Hasan Sadikin General Hospital is only by performing a spirometry examination without health status assessment similar to the recommendation of the Global Initiative for Chronic Obstructive Lung Disease (GOLD), although those tools assess different things.

Correspondence: Dian Nuryanda, Faculty of Medicine, Universitas Padjadjaran, Jalan Raya Bandung-Sumedang Km.21, Jatinangor, Sumedang, Indonesia, Email: diannuryanda@yahoo.com 
The aim of this study was to determine the relationship between pulmonary function examination represented by FEV1 with CAT and MMRC dyspnea scale scoring among COPD patients.

\section{Methods}

The population of this study is patients with confirmed COPD. Samples were obtained from medical records of patients visiting the Pulmonary Clinic, Internal Medicine Department, at Hasan Sadikin General Hospital, Bandung. Data were taken after the approval from Hasan Sadikin General Hospital
Ethics Committee was obtained. Data were taken to fulfill the minimum size of sample. This study was conducted in the Department of Internal Medicine, Respirology Sub Division on September-October 2014. The sampling method used in this study was purposive sampling. Moreover, the inclusion criteria were patients with confirmed diagnosis COPD and complete medical records, containing the observed variables, FEV1 measurement result, CAT and MMRC scoring results. Patients who had asthma as comorbid and FEV1/Forced Volume Capacity (FVC) $\geq 70 \%$ were excluded from this study. Out of 80 samples that were included in this study, 3 of them were excluded.

Table 1 Characteristic of the Subjects

\begin{tabular}{|c|c|}
\hline Characteristic & $\mathrm{n}=77$ \\
\hline Age (year) & $69(50-89)$ \\
\hline \multicolumn{2}{|l|}{ Gender } \\
\hline Male & $56(72.7)$ \\
\hline Female & $21(27.3)$ \\
\hline \multicolumn{2}{|l|}{ Spirometry Measurement Results (\%) } \\
\hline FEV1 pre-bronchodilator & $48.30 \pm 11.6$ \\
\hline FVC pre-bronchodilator & $60.58 \pm 13.2$ \\
\hline FEV1 post-bronchodilator & $52.5 \pm 11.8$ \\
\hline FVC post-bronchodilator & $63.54 \pm 13.1$ \\
\hline FEV1/FVC pre-bronchodilator & $63.6(47.4-69)$ \\
\hline FEV1/FVC post bronchodilator & $66.7(49.3-69.9)$ \\
\hline \multicolumn{2}{|l|}{ Spirometry Classification } \\
\hline GOLD 1 & $1(1.3 \%)$ \\
\hline GOLD 2 & $43(55.8 \%)$ \\
\hline GOLD 3 & $33(43.9 \%)$ \\
\hline GOLD 4 & 0 \\
\hline \multicolumn{2}{|l|}{ MMRC dyspnea scale } \\
\hline 1 & $20(26 \%)$ \\
\hline 2 & $26(22.38 \%)$ \\
\hline 3 & $24(31.2 \%)$ \\
\hline 4 & $7(9.1 \%)$ \\
\hline 5 & 0 \\
\hline \multicolumn{2}{|l|}{ CAT } \\
\hline$\leq 10$ & 0 \\
\hline $11-20$ & $27(35.1 \%)$ \\
\hline $21-30$ & $42(54.5 \%)$ \\
\hline$>30$ & $8(10.4 \%)$ \\
\hline
\end{tabular}


This was an observational analytical study with a cross sectional design. The data were analyzed using a statistic analytic computer program. Normality of the data was tested using Kolmogorov-Smirnov Test. In addition, Pearson's Correlation Test was used to analyze correlation of parametric variable, while Spearman's Correlation Test was used to analyze nonparametric variable.

\section{Results}

Based on characteristic of the subjects, the age of subjects ranged from 50-89 years, and males were more common than females. Spirometry assessment result showed that most of the subjects were in moderate-severe classification based on GOLD. While, MMRC dyspnea scale scoring result of the subjects waswere ranged from 1-4, and for CAT was ranged from 12 for the minimum score, and 35 for the maximum score (Table 1).

Moreover, Pearson's test was performed on FEV1 post bronchodilator administration and CAT scoring. Results of the analysis showed that there was a significant relationship between the two variables with $\mathrm{p}=0.035(\mathrm{p}<0.05)$. A poor correlation between the two variables is indicated by the value of the correlation coefficient (r) of 0.24 . The direction of the correlation showed the inverse relationship between the two variables. In the event of an increase in CAT scoring result, a decline in the value of FEV1 post bronchodilator can be observed.

Spearman's test was performed on FEV1 post bronchodilator and scoring results were obtained using MMRC dyspnea scale. The data showed different results from the Pearson's test. While the correlation test showed no significant relationship between the two variables, indicated with $\mathrm{p}=0.198$ and the value of the correlation coefficient (r) 0.148 ( $p>0.05)$.

\section{Discussion}

Previous studies that discussed the relationship between the health status of
COPD patients with FEV1 assessment results provide variations in results, starting from a weak correlation and insignificant to a strong correlation and significant. ${ }^{5-9}$ The results in this study found that CAT had a significant but weak correlation with FEV1 post bronchodilator administration, while MMRC did not provide a significant relationship.

Similar results were obtained in the study conducted by Garrido et al. ${ }^{6}$, which mentioned that the decline in FEV1 measurement results are significantly correlated $(\mathrm{r}=0.38 ; \mathrm{p}<0.001)$ with a reduced quality of life in COPD patients assessed by the Physical Component Score (PCS). Garrido et al. ${ }^{6}$ also found that FEV1 post bronchodilator administration is related to the Mental Component Score (MCS), but with a weak correlation $(\mathrm{r}=0.12)$. The result of this study suggested a relationship between the degrees of airflow limitation that is indicated by a decrease in FEV1 post bronchodilator administration with the patient's quality of life. The severity of COPD affects health status of patients, both mentally and physically.

Furthermore, Tsilligiani et al. ${ }^{7}$ conducted a similar study analyzing the FEV1 post bronchodilator administration with the COPD patient's health status. Questionnaires were used to assess the health status in this study, including St. George Respiratory Questionnaire (SGRQ), COPD Control Questionnaire (CCQ), the Quality of Well Being (QWB) scale, as well as the Chronic Respiratory Questionnaire (CRQ). ${ }^{7}$ In this study, both CAT and MMRC dyspnea scale were not used.

The study by Tsilligiani et al. ${ }^{7}$ supported the results of this study. The questionnaire that provides the most robust correlation to FEV1 is shown by QWB, CCQ and CRQ while the correlation shown by the other questionnaire were poor. ${ }^{7}$ This is due to the use of different questionnaires .

In addition, Yeo et al. $^{8}$ conducted a study that conflicted with the results of this study. Yeo et $a .^{8}$ stated that there is no significant correlation between the disease severities based on GOLD staging represented by the percentage of FEV1 post bronchodilator administration with health status questionnaire assessed using SGRQ. Besides,

Table 2 Data Analysis Result

\begin{tabular}{lcc}
\hline & \multicolumn{2}{c}{ FEV1 post-bronchodilator } \\
\cline { 2 - 3 } & Sig. (2-tailed) & Correlations Coefficient (r) \\
\hline CAT & 0.035 & -0.240 \\
MMRC & 0.198 & -0.148 \\
\hline
\end{tabular}


Yeo et al. $^{8}$ mentioned that there is a tendency in which the patients with more severe illness have poorer health status, but there is no significant difference between patient's health status and the disease severity. This is due to COPD patients may experience a wide range of declining health status, independent to the severity of the disease.

A research conducted by Voll-Aanerud et al. ${ }^{9}$ regarding the association of symptoms and the severity of COPD respiration on Health Related Quality of Life (HRQoL) using SF-12 questionnaire showed similar results. The SF-12 questionnaire assessed HRQoL with 2 components, Physical Component Score (PCS) and Mental Component Score (MCS). A rise in the PCS and MCS shows a better HRQoL. Results obtained from this study are the PCS and MCS scoring result were more significantly associated with respiratory symptoms compared to the severity of COPD. ${ }^{9}$

Previous literatures found that the FEV1 measurement result only shows a lung function abnormalities without being able to assess the health status of a COPD patients. ${ }^{1,7,9,10}$ In this study, the MMRC dyspnea scale did not significantly correlate with decreasing FEV1 post bronchodilator administration. It could be said that the health status assessed by the MRC dyspnea scale, which is namely the limitation in performing daily activities and possible risks of exacerbation, did not have a significant correlation with the disease severity measured by FEV1 post bronchodilator administration. This happens due to the etiology of shortness of breath or disability arises not only because of the obstruction of the respiratory tract, so that in the event of a declining lung function did not significantly occur also with shortness of breath. Breathlessness in patients with COPD is a typical symptom. ${ }^{7}$ A declining FEV1 post bronchodilator were not associated with the risk of exacerbations of COPD patients.

Study that conducted by Pitta et $\mathrm{al}^{5}$ showed that reduction in daily activities of COPD patients does not depend on the disease severity determined only by the measurement of FEV1. Although the declined FEV1 is indicated to the deterioritation of airflow limitation, but it should not be used by itself to rule out the disease severity. ${ }^{5-7}$

Moreover, GOLD mentioned that in each respective GOLD category, the health status of a patient with COPD can vary from patients with good health status to poor health status. ${ }^{1}$ This could be the influencing factors to the correlations between the disease severity which is indicated by the results of measurements of FEV1 post bronchodilator administration with both CAT and MMRC dyspnea scale, as discussed by Yeo et al. ${ }^{8}$

As previous studies mentioned before, it can be concluded that the dissimilarities in the results of this study is also influenced by other factors such as gender, age, comorbid disease, and the patient's condition at the time of examination; stable COPD patients give different test results with patients who are experiencing an exacerbation. An important goal that must be achieved in the health status assessment of patients with COPD is the provision of both pharmacological and non-pharmacological treatment according to the needs of the patient. Routine examination of patients with COPD by spirometer is not sufficient to assess the health status of the patient. GOLD mentioned that the assessment of COPD patients should include a separate assessment of the 4 following, namely the risk of exacerbations, symptoms experienced by patients at this time, abnormality in spirometry measurement results, and comorbids experienced by the patient. ${ }^{1}$

The limitation of this study was using the cross sectional study design; this method is considered to have a greater likelihood of bias. The available data show no information of comorbids suffered by the patients and the patients' condition during examination, whether they were in a stable condition or were experiencing exacerbations, as these things can be a confounding factor to this study. The number of samples and the age were not normally distributed; which is also considered as a limitation in this study.

By understanding the relationship between health status assessments and spirometry measurements results shown in this study, it is expected that the process of examination and assessment of COPD patients in all health facilities can be performed comprehensively in accordance with the recommendations given by GOLD.

\section{References}

1. Vestbo J, Hurd S, Agusti A, Jones P, Vogelmeier C, Anzueto A, et al. Global strategy for the diagnosis, management, and prevention of chronic obstructive pulmonary disease: GOLD executive summary. Am J Respir Crit Care Med. 2014;187(4):347-65.

2. WHO. Global status report on noncommunicable diseases 2010: description of the global burden of NCDs, 
their risk factors and determinants. Geneva: WHO Library Cataloguing-inPublication Data; 2011.

3. Jones P, Harding G, Berry P, Wiklund I, Chen W, Kline Leidy N. Development and first validation of the COPD Assessment Test. Eur Respir J. 2009;34(3):648-54.

4. Jones PW, Nadeau G, Small M, Adamek L. Characteristics of a COPD population categorised using the GOLD framework by health status and exacerbations. Respir Med. 2014;108(1):129-35.

5. Pitta F, Takaki MY, Oliveira NHD, Sant'Anna TJP, Fontana AD, Kovelis D, etal. Relationship between pulmonary function and physical activity in daily life in patients with COPD. Respir Med. 2008;102(8):1203-7.

6. Garrido PC, Diez JDM, Gutierrez JR, Centeno AM, Vazquez GE, Angel GDM, et al. Negative impact of chronic obstructive pulmonary disease on the health-related quality of life of patients. Results of the EPIDEPOC study. Health Qual Life Outcomes. 2006;4:31:1-9.
7. Tsiligianni I, Kocks J, Tzanakis N, Siafakas $\mathrm{N}$, van der Molen T. Factors that influence disease-specific quality of life or health status in patients with COPD: a review and meta-analysis of Pearson correlations. Prim Care Respir J. 2011;20(3):257-68.

8. Yeo J, Karimova G, Bansal S. Co-morbidity in older patients with COPD-its impact on health service utilisation and quality of life, a community study. Age Ageing. 2006;35(1):33-7.

9. Voll-Aanerud M, Eagan TM, WentzelLarsen T, Gulsvik A, Bakke PS. Respiratory symptoms, COPD severity, and health related quality of life in a general population sample. Respir Med. 2008;102(3):399406.

10. Wise RA. Chronic obstructive pulmonary disease: clinical course and management. In: Fishman AP, Elias JA, Fishman JA, Grippi MA, Senior RM, Pack AI, editors. Fishman's pulmonary diseases and disorders. $4^{\text {th }}$ ed. New York: Mc Graw Hill; 2008. p. 729-46. 